\title{
Genetic embedded matching approach to ground states in continuous-spin systems
}

\author{
Martin Weige ${ }^{*}$ \\ Department of Mathematics and the Maxwell Institute for Mathematical Sciences, \\ Heriot-Watt University, Edinburgh, EH14 4AS, UK and \\ Department of Physics and Astronomy, University of Waterloo, Waterloo, Ontario, N2L 3G1, Canada
}

(Dated: October 30, 2018)

\begin{abstract}
Due to an extremely rugged structure of the free energy landscape, the determination of spinglass ground states is among the hardest known optimization problems, found to be $\mathcal{N} \mathcal{P}$-hard in the most general case. Owing to the specific structure of local (free) energy minima, general-purpose optimization strategies perform relatively poorly on these problems, and a number of specially tailored optimization techniques have been developed in particular for the Ising spin glass and similar discrete systems. Here, an efficient optimization heuristic for the much less discussed case of continuous spins is introduced, based on the combination of an embedding of Ising spins into the continuous rotators and an appropriate variant of a genetic algorithm. Statistical techniques for insuring high reliability in finding (numerically) exact ground states are discussed, and the method is benchmarked against the simulated annealing approach.
\end{abstract}

PACS numbers: 75.50.Lk, 02.60.Pn, 75.10.Hk

\section{INTRODUCTION}

Complex (free) energy landscapes featuring a multitude of local minima separated by energy barriers are common in problems of statistical mechanics, chemical and biophysics consequently often subsumed under the label of "complex systems", be it biopolymers, structural or spin glasses [1]. The consequences of this deviation from the classical textbook situation of a potential energy with at most a handful of metastable states are dramatic for the static and dynamic behavior of the affected systems in nature, including for instance "memory" and "rejuvenation" effects in spin glasses [2], but no less pronounced for the theoretical investigation of models of such situations with computational simulation or optimization methods: here, model systems get trapped in local minima for exponentially long times, preventing an equilibration in finite-temperature simulations [2] or lead to a vastly increased effort needed for an optimization procedure to yield ground states with finite probability [3]. Clearly, the presence of many local minima alone is not sufficient to pose serious problems for any optimization method more elaborate than a strictly downhill, local search. Rather, it is the organization of minima and interjacent barriers that is the cause for the trapping phenomena, and distinguishes the milder from the more severe cases [1]. While, for instance, many typical biopolymers exhibit landscapes with moderate barriers separating minima of substantially different energies, with a "funneling" towards a unique global minimum [4], disordered and frustrated magnetic systems are rather characterized by many (quasi-) degenerate minima close

*Present address: Institut für Physik, Johannes-GutenbergUniversität Mainz, Staudinger Weg 7, 55099 Mainz, Germany; Electronic address: weigel@uni-mainz.de to the ground state(s) separated by large barriers, leading to much more severe effects of metastability and slow relaxation [5, $[6]$.

Independent of this connection between the structure of the energy landscape and the real or artificial (Monte Carlo or optimization) dynamics of a frustrated system, the problem of finding ground states or, alternatively, partition functions, of the corresponding models has been considered as a problem in the field of computational complexity 7]. In computer science, traditionally mostly the "worst case" complexities of algorithms have been considered, i.e., the asymptotic scaling of the run time $T(N)$ with the problem size $N$ for the "hardest" set of input data within the class of allowed inputs, $T_{\max }(N)$ [8]. Quite generally, problems with an asymptotically polynomial form of $T_{\max }(N)$ are considered tractable, whereas an exponential divergence for the best known algorithm is associated with intractability. Paradigmatic results have been found for decision problems with "yes" or "no" answers, for which a powerful classification scheme has been established: problems with a known polynomial algorithm are grouped in $\mathcal{P}$, whereas a more general class of problems for which the correctness of a solution can be checked in polynomial time is denoted $\mathcal{N} \mathcal{P}$. The potential hardness of the latter must then exclusively result from the exponential growth of the search space, such that a theoretical computer capable of infinite parallelism can solve such problems in polynomial time [7]. The hardest $\mathcal{N} \mathcal{P}$ problems, namely those whose polynomial solution would imply polynomial complexity for all other $\mathcal{N} \mathcal{P}$ problems, are termed $\mathcal{N} \mathcal{P}$ complete, which includes most of the well-known hard problems such as the traveling salesman problem or the satisfiability problem. While it is possible that all such problems might have polynomial-time solutions, i.e., $\mathcal{P}=\mathcal{N} \mathcal{P}$, this is now considered to be extremely unlikely, and $\mathcal{N} \mathcal{P}$ problems almost certainly require an exponential computational effort [8]. This classification extends to optimiza- 
tion (instead of decision) problems where, specifically, those where the problem of deciding about the existence of a solution better than a given bound in the cost function is $\mathcal{N P}$ complete, are termed $\mathcal{N P}$ hard.

For the Ising spin glass with Hamiltonian [9]

$$
\mathcal{H}=-\sum_{\langle i j\rangle} J_{i j} \boldsymbol{S}_{i} \cdot \boldsymbol{S}_{j},
$$

the problems of computing ground states or the partition function are known to be $\mathcal{N P}$ hard in space dimensions $d \geq 3$ or for two-dimensional (2D) systems in a magnetic field [10, 11]. The zero-field 2D problem, on the other hand, is tractable in polynomial time [12, 13, 14]. In particular, ground states on planar graphs can be found by means of the mapping to a minimum-weight perfect matching problem, as discussed below in Sec. II While thus, generically, spin-glass ground-state problems are hard, one has to keep in mind that this classification concerns the worst-case behavior among all possible realizations of couplings $J_{i j}$ of the chosen distribution (e.g., bimodal or Gaussian), whereas it is, for instance, simple to specify the ground state of the purely ferromagnetic system with $J_{i j}=J_{0}>0$, which also belongs to the allowed $J_{i j}$ realizations. Hence, relevant for actual computations is also the average complexity, depending on a chosen probability distribution $P\left(\left\{J_{i j}\right\}\right)$ of couplings. Within the spin-glass phase, however, also this mean complexity is exponential for known exact approaches to the problem in $d>2$ [15]. Correspondingly, heuristic optimization techniques for finding low-lying or ground states are called for. These might include generic approaches, such as simulated annealing [16], multicanonical [17] or parallel tempering [18] Monte Carlo simulations, but also a number of methods specifically tailored to the problem $19,20,21,22,23$, the latter generally showing the best performance [3, 24]. Insofar as these methods make use of some type of relaxational (quasi-) dynamics, they to some extent also suffer from the slowness of relaxation entailed by the structure of free-energy minima and separating barriers. It should be pointed out, however, that such slow dynamics is not equivalent to hardness in the classifications of computational complexity [25]. Instead, slower than power-law relaxation of local dynamics also occurs in computationally polynomial systems [5], such as the Ising spin-glass model in two dimensions [12]. The stochastic nature of most of these approaches requires a different description of their time complexity or efficiency: since such methods do not guarantee to yield ground states, one should now rather ask for the worst case or mean computational effort to end up in a ground state with an a priori prescribed success probability $p_{s}$ (for $p_{s}=0.95$, say), or for the distribution (over disorder realizations) of such minimal running times at fixed $p_{s}$. A framework for such considerations will be developed below in Sec. IV] Ground-state searches for spin-glass systems are additionally complicated by an extraordinarily broad distribution of "hardness" over disorder samples, which draws into question the treatment of all samples with constant computational effort. In this context, it is discussed below in how far properties of individual disorder samples can serve as hardness indicators and hence an automatic effort adaptation can be achieved.

Ising spin-glass ground states have been considered with the aim to understand the nature of the lowtemperature phase while avoiding the equilibration problems of finite-temperature simulations. Ground-state computations for systems with different boundary conditions or with some fixed spins allow for the direct investigation of domain-wall and droplet defects, whose properties should reveal in how far finite-dimensional spin glasses are correctly described by mean-field theory (see Ref. 26] for a review of recent developments). The polynomially tractable 2D case, in particular, has provided a fruitful playground for testing theoretical pictures of the spin-glass phase, and remains a topic of active research to date [27, 28, 29, 30]. In terms of spin-glass phases realized experimentally, in particular in the multitude of systems with frustrating lattice structures that have come into focus more recently [31], systems with continuous spins are probably more common than the extremely anisotropic Ising case. In computing ground states for such systems, modeled, say, by the EdwardsAnderson Hamiltonian (1) with continuous $\mathrm{O}(n)$ spins $\boldsymbol{S}_{i}$, one leaves the relatively well-understood field of combinatorial optimization. To my understanding, nothing is known about the (suitably generalized) computational complexity of this problem. It is easily seen [3, 8], however, that already the $q$-state Potts spin glass corresponds to a multi-terminal flow problem known to be $\mathcal{N P}$ hard even in two dimensions for $q=3$ [32]. Nothing would seem to indicate that the $X Y$ case of continuous planar spins, or the Heisenberg model of $\mathrm{O}(3)$ rotators could be easier computationally than the discrete Potts approximation. With the exception of a study of the $X Y$ spin glass in the Coulomb gas representation [33], all studies of low-lying metastable states in $\mathrm{O}(n)$ spin glasses (with $n>1$ ) have relied on variants of a simple spin-quench technique corresponding to a $T=0$ Monte Carlo simulation with local updates [34, 35, 36, 37] (apart from studies of the computationally simpler case of the $n \rightarrow \infty$ spherical spin glass [38]). This spin quench follows from noting that a necessary condition for metastability is that each spin be parallel to its local molecular field,

$$
\boldsymbol{S}_{i} \| \boldsymbol{h}_{i}=\sum_{j} J_{i j} \boldsymbol{S}_{j}
$$

leading to the prescription of an iterative alignment of single spins $\boldsymbol{S}_{i}$ parallel to $\boldsymbol{h}_{i}$. In contrast to the investigations of the Ising spin glass, none of these approaches have allowed to find numerically exact ground states with a reasonably high probability, such that, instead, effectively the properties of some set of metastable states with unclear relation to the ground-state behavior have been found and investigated. To improve on this, it is proposed here to combine exact ground-state computations of Ising variables embedded into the continuous spins with a spe- 
cially tailored genetic algorithm exploiting the locally rigid cluster structure of metastable spin configurations [39]. This results in an efficient approach for groundstate computations of continuous-spin systems on planar graphs, which is tested and assessed here for the case of the bimodal $X Y$ spin glass on the square lattice, where numerically exact ground states can be found with high reliability for systems up to about $30 \times 30$ spins using currently available computational resources. Implications of these results for the nature of the low-temperature phase of this model have been discussed elsewhere [39, 40].

The rest of the paper is organized as follows. Section II is devoted to a description of the embedded matching technique for continuous spins, while in Sec. III the combination of this approach with a genetic algorithm with cluster exchange is discussed. In Sec. IV the performance of this approach for the 2D $X Y$ spin glass is investigated in terms of a detailed statistical analysis, focusing on the large variations between disorder replica and offering a standardized approach of "quality assurance" for stochastic optimization algorithms. An exhaustive benchmarking of the new approach against generalpurpose techniques is not feasible. At least, however, results comparing to the simple spin-quench used before and a more elaborate simulated annealing approach are presented in some detail. Finally, Sec. V contains my conclusions.

\section{EMBEDDED MATCHING}

For attacking the ground-state problem of continuous spin glasses in two dimensions, inspiration is taken from the polynomial solution of the Ising problem, which is hence described first, and then adapted to continuous spins via an embedding of Ising variables.

\section{A. Ising ground states as perfect matchings}

The polynomial complexity of the 2D Ising spin glass allows for the formulation of efficient algorithms for finding ground states and computing the partition function. A number of different techniques has been established for the calculation of the latter [10, 13, 14], mostly relying on the computation of Pfaffians, but these will not be needed here. Computations of ground states rest on the concept of frustrated loops introduced by Toulouse [41]: in the presence of couplings $J_{i j}$ of either sign, for each closed curve along lattice links touching an odd number of antiferromagnetic bonds $\left(J_{i j}<0\right)$, one cannot find a spin configuration satisfying all pair interactions, i.e., $J_{i j} S_{i} S_{j}<0$ for at least one ("broken") edge. Hence, the presence of such loops is responsible for the excess of the ground-state energy of the spin glass above the unfrustrated value $E_{\mathrm{FM}}=-\sum_{\langle i j\rangle}\left|J_{i j}\right|$. Due to the contractibility of all loops on a planar graph, in this case it suffices to concentrate on the frustration of the plaque-

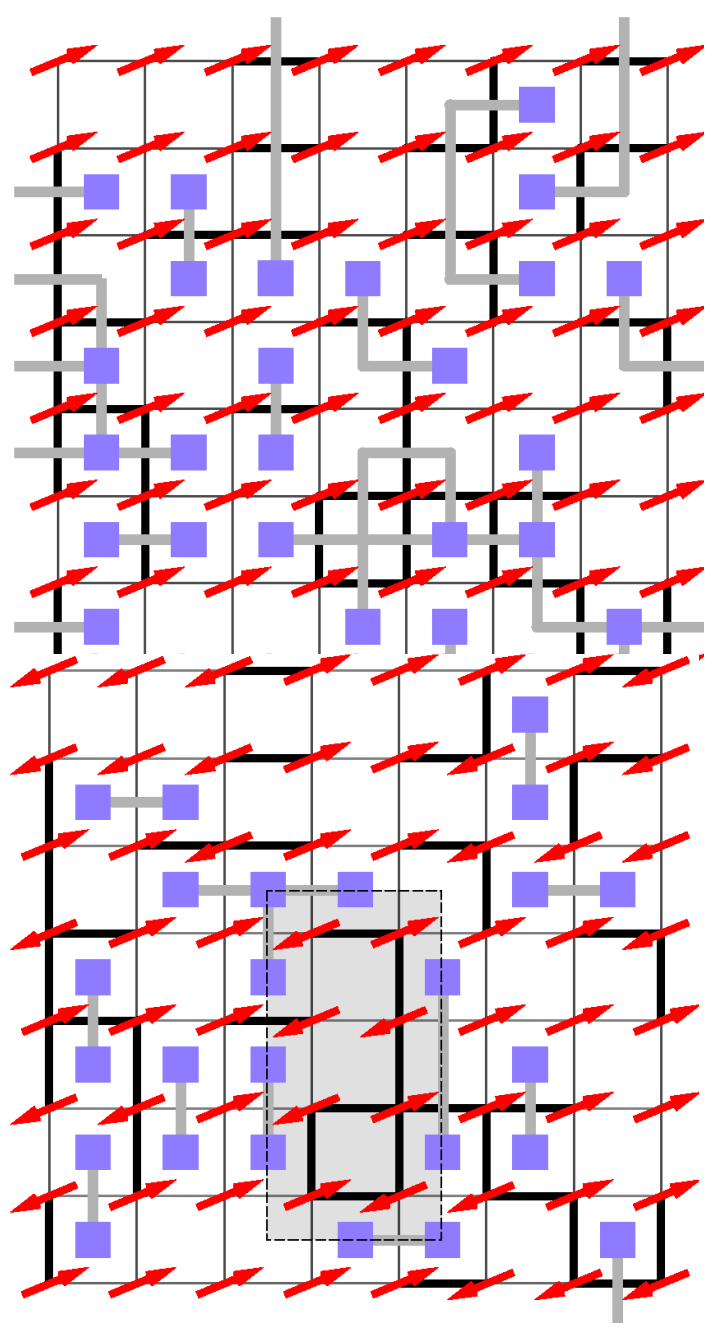

FIG. 1: (Color online) Transformation of the Ising groundstate calculation on the square lattice to a matching problem. Upper panel: Frustrated plaquettes (marked by small squares) have an odd number of antiferromagnetic (bold) bonds. The set of broken bonds forms a collection of lines on the dual lattice (shaded, gray lines). Lower panel: a ground state of the system is given by a minimum-weight perfect matching of frustrated plaquettes. The dashed line indicates an alternating cycle along which an exchange of matched and unmatched edges yields another perfect matching.

ttes, i.e., the elementary faces of the lattice [12]. This is illustrated by the marking of frustrated plaquettes for the square lattice in Fig. 1. For each plaquette $\square_{n}$, define the frustration function [41]

$$
\Phi_{\square_{n}}=\prod_{(i, j) \in \square_{n}} \operatorname{sign} J_{i j}= \pm 1,
$$

such that $\Phi_{\square_{n}}=-1$ if and only if $\square_{n}$ is frustrated. By this definition, in a configuration of the Ising spins each frustrated plaquette must have an odd number (1 or 3 for the square lattice) of broken bonds, whereas an unfrustrated plaquette is surrounded by an even number of broken bonds (0,2 or 4 for the square lattice). Bonds drawn dual to the broken bonds then connect to form 
energy strings starting and terminating in frustrated plaquettes, cf. the sketch in the upper panel of Fig. 1. The excess energy is

$$
\frac{1}{2}\left(E-E_{\mathrm{FM}}\right)=W_{\text {string }}=\sum_{\text {strings }}\left|J_{i j}\right|,
$$

and a ground state corresponds to a collection of strings

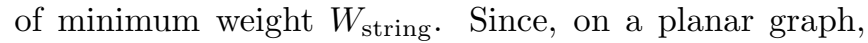
closed loops of dual bonds can be contracted away, they cannot occur in a ground state, which hence corresponds to a minimum-weight perfect matching of the frustrated plaquettes. This is illustrated in the lower panel of Fig. 1. The planarity of the lattice ensures that each such matching corresponds to a valid spin configuration [12].

Following the above discussion, this matching problem is defined on the complete graph $\mathcal{G}=\mathcal{F} \times \mathcal{F}$ on the set $\mathcal{F}$ of frustrated plaquettes. Each of the $|\mathcal{E}|=|\mathcal{F}||\mathcal{F}-1|$ edges $e_{m n}=\left(f_{m}, f_{n}\right)$ carries a weight

$$
W\left(e_{m n}\right)=\min _{\gamma_{m n}} \sum_{(i, j) \in \gamma_{m n}}\left|J_{i j}\right|,
$$

corresponding to the minimum weight of all paths $\gamma_{m n}$ on the (original) lattice connecting the plaquettes $f_{m}$ and $f_{n}$. Hence an auxiliary minimum-cost path problem needs to be solved as an input to the matching calculation. This is most efficiently achieved by an appropriate implementation of Dijkstra's algorithm with $\mathrm{O}(|\mathcal{E}| \ln |\mathcal{E}|)$ complexity, or, for the case of a bimodal $\mathrm{P}\left(J_{i j}\right)$ where $\left|J_{i j}\right|=J_{0}$ for all edges, by a simple breadth-first search [42]. Since there is an even number of frustrated plaquettes [78], a perfect matching on $\mathcal{G}$ can always be found. A polynomial algorithm for the matching problem on general graphs has been proposed by Edmonds [43]. It proceeds by successively identifying augmenting paths in the matching graph, i.e., cycles of alternating matched and unmatched edges such that an exchange matched $\leftrightarrow$ unmatched decreases the overall weight. This is illustrated by a cycle in the original lattice in the lower panel of Fig. 1. The complexity of the original implementation is $\mathrm{O}\left(|\mathcal{F}|^{2}|\mathcal{E}|\right)$. The present implementation is based on the "Blossom IV" matching algorithm of Cook and Rohe incorporating many improvements developed in the combinatorial optimization literature after Edmonds' original proposal [4].

Given a solution to the matching problem, a corresponding spin configuration is found by arbitrarily choosing the orientation of one spin and successively implementing the satisfaction constraints expressed by the perfect matching via selecting spin orientations in a breadthfirst search emanating from the chosen starting point. Note that, depending on the distribution of couplings $J_{i j}$, neither the solution of the matching problem nor the mapping back to spin configurations needs to be unique [45]: if some edges in the matching problem have the same weight, there could be different matchings of minimum weight. On the other hand, also the notion of minimum-weight paths on the original square lattice is

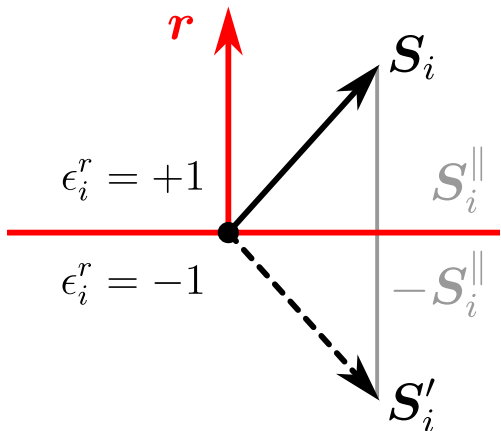

FIG. 2: (Color online) Embedding of Ising spins into the continuous rotators $\boldsymbol{S}_{i}$ via decomposition with respect to a direction $\boldsymbol{r}$ in spin space.

not necessarily unique, and more than one path between two frustrated plaquettes could be of minimal weight. Finally, each configuration of energy strings corresponds to two different spin states, related by spin inversion. This generally leads to a large ground-state degeneracy for discrete and rational distributions $P\left(\left\{J_{i j}\right\}\right)$, but a unique ground state, e.g., for the Gaussian case [46]. For the complete graph $\mathcal{G}$, the time complexity of Edmonds' implementation would be $\mathrm{O}\left(|\mathcal{F}|^{4}\right)$, corresponding to $\mathrm{O}\left(L^{8}\right)$ for a $L \times L$ lattice. Although this is polynomial, further improvements are highly desirable to reduce the rather large exponent and enable treatment of reasonably sized problem instances. This can be achieved by a thinning of the complete graph $\mathcal{G}$ : a matching of frustrated plaquettes in the ground state becomes more and more unlikely with increasing weight of the path connecting them, and such large-weight edges can consequently be disregarded. Suitable cutoff parameters depend on the distribution of $J_{i j}$, and have to be tested thoroughly. For the implementation used here, cutoffs at fixed maximum path weight and conditions on the minimum vertex degree in the matching graph have been employed with comparable success.

\section{B. Embedded matching for continuous spins}

For continuous spins, the notion of plaquette frustration stays meaningful, since it is a property of the bond configuration only. The transformation to a matching problem, however, is restricted to discrete Ising spins. To leverage the tractability of the Ising case for the treatment of continuous-spin systems, an embedding of Ising spins into the continuous rotators is employed. To this end, consider an arbitrary direction $\boldsymbol{r},|\boldsymbol{r}|=1$, in spin space common to all lattice sites, and decompose the $\mathrm{O}(n)$ spins $\boldsymbol{S}_{i}$ of Eq. (11) as $\boldsymbol{S}_{i}=\boldsymbol{S}_{i}^{\|}+\boldsymbol{S}_{i}^{\perp}=\left(\boldsymbol{S}_{i} \cdot \boldsymbol{r}\right) \boldsymbol{r}+\boldsymbol{S}_{i}^{\perp}$, cf. the illustration in Fig. 2, This induces a decomposition $\mathcal{H}=\mathcal{H}^{r, \|}+\mathcal{H}^{r, \perp}$ with

$$
\mathcal{H}^{r, \|}=-\sum_{\langle i, j\rangle} \tilde{J}_{i j}^{r} \epsilon_{i}^{r} \epsilon_{j}^{r},
$$


where $\epsilon_{i}^{r}=\operatorname{sign}\left(\boldsymbol{S}_{i} \cdot \boldsymbol{r}\right)$, and where the effective couplings $\tilde{J}_{i j}^{r}$ are given by

$$
\tilde{J}_{i j}^{r}=J_{i j}\left|\boldsymbol{S}_{i} \cdot \boldsymbol{r} \| \boldsymbol{S}_{j} \cdot \boldsymbol{r}\right| .
$$

Hence, with respect to reflections of the spins $\boldsymbol{S}_{i}$ along the plane defined by $\boldsymbol{r}$, the signs $\epsilon_{i}^{r}$ are Ising variables (cf. Fig. 22), and the embedded Hamiltonian (6) is that of an Ising model. Note that with respect to these reflections $\boldsymbol{S}_{i} \mapsto \boldsymbol{S}_{i}-2\left(\boldsymbol{S}_{i} \cdot \boldsymbol{r}\right) \boldsymbol{r}$, the perpendicular part $\mathcal{H}^{r, \perp}$ is invariant and thus does not contribute to the embedded dynamics. A similar embedding of Ising variables has been used to formulate a cluster-update Monte Carlo algorithm for continuous spins [47].

Updating the effective Ising variables $\epsilon_{i}^{r} \mapsto-\epsilon_{i}^{r}$ via plane reflections $\boldsymbol{S}_{i} \mapsto \boldsymbol{S}_{i}-2\left(\boldsymbol{S}_{i} \cdot \boldsymbol{r}\right) \boldsymbol{r}$, a ground state of the Hamiltonian $\mathcal{H}^{r, \|}$ of (6) can be found, for instance, using the transformation to a matching problem outlined above. Note that since sign $\tilde{J}_{i j}^{r}=\operatorname{sign} J_{i j}$ according to Eq. (7), the frustration function $\Phi_{\square_{n}}$ does not depend on the embedding direction $\boldsymbol{r}$, and only the weights of the matching graph $\mathcal{G}$ must be updated for each embedded matching computation. The rotational symmetry of the $\mathrm{O}(n)$ Hamiltonian (11) can then be recovered by a random sampling over different embedding directions $\boldsymbol{r}$. This leads to the following algorithm:

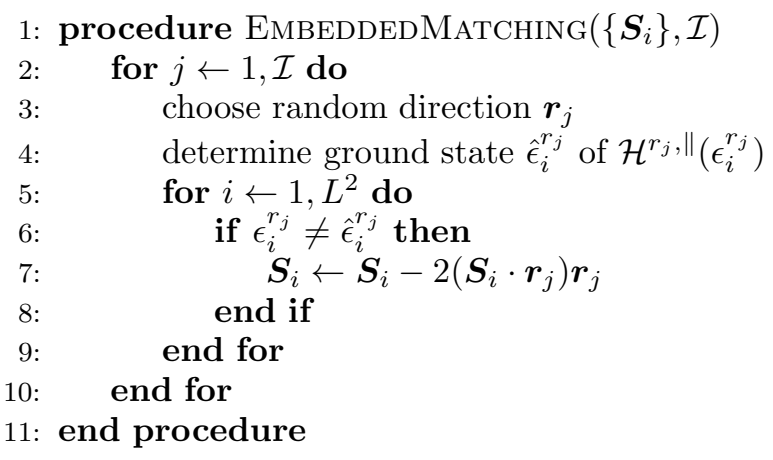

For each direction $\boldsymbol{r}$, it holds that $\mathcal{H}=\mathcal{H}^{r, \|}\left(\left\{\epsilon_{i}^{r}\right\}\right)+$ $\mathcal{H}^{r, \perp} \geq \mathcal{H}^{r, \|}\left(\left\{\hat{\epsilon}_{i}^{r}\right\}\right)+\mathcal{H}^{r, \perp}$, where $\hat{\epsilon}_{i}^{r}$ is the ground state configuration of the embedded Ising model. Consequently, the embedded matching procedure corresponds to a strictly downhill minimization approach. If $\mathcal{H}$ is in a ground state, $\mathcal{H}^{r, \|}\left(\left\{\epsilon_{i}^{r}\right\}\right)=\mathcal{H}-\mathcal{H}^{r, \perp}$ must be in a ground state for each $\boldsymbol{r}$ as well. Conversely, however, $\mathcal{H}^{r, \|}\left(\left\{\epsilon_{i}^{r}\right\}\right)$ being in a ground state for each $\boldsymbol{r}$ does not guarantee global minimum energy for the full $\mathcal{H}$. This is due to the fact that the embedded couplings $\tilde{J}_{i j}^{r}$ of (7) depend on the spin configuration $\left\{\boldsymbol{S}_{i}\right\}$ and hence on the history of previous embedding matching runs. As a consequence, the dynamics of $\left\{\boldsymbol{S}_{i}\right\}$ induced by the embedded matching procedure has metastable states [79]. The number of metastable states is far less, however, than for the local spin-quench approach of Eq. (2), since for each direction $\boldsymbol{r}$ a global minimum is found: while $\mathcal{H}^{r_{i}, \|}$ converges to a locally spin-flip stable state (otherwise a direction $\boldsymbol{r}$ could be found, for which embedded Ising minimization would lead to reflections of one or more spins), not every such

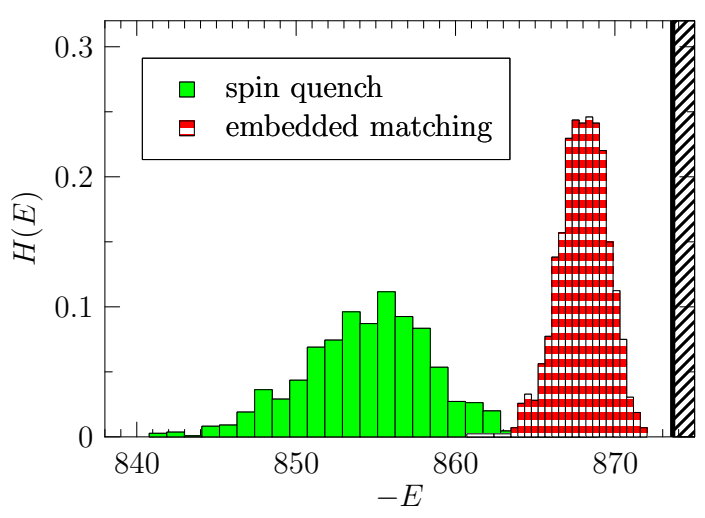

FIG. 3: (Color online) Histograms of energies of metastable states of a $24 \times 24$ sample of the $\pm J X Y$ spin glass on the square lattice as found from 1000 independent runs of the embedded matching technique compared to results of a local spin quench. The onset of the hatched area to the right indicates the ground-state energy of this sample.

locally stable state is metastable with respect to the embedded matching procedure (because the embedded Ising system for some direction $\boldsymbol{r}$ could be metastable instead of globally optimal).

It is found numerically that the sequence $\left\{E_{i}\right\}$ of energies of the embedded matching approach always converges. As a consequence of the dependency of $\tilde{J}_{i j}^{r}$ of (7) on $\left\{\boldsymbol{S}_{i}\right\}$, the limit $E_{\infty}$ depends on the particular sequence $\left\{\boldsymbol{r}_{1}, \boldsymbol{r}_{2}, \ldots\right\}$ of chosen directions. Figure 3 shows a histogram of energies found from the embedded matching approach with a number of different random starting configurations and different series of embedding directions for a particular $24 \times 24$ sample of the $2 \mathrm{D}$ bimodal $X Y$ spin glass. For comparison, a corresponding histogram for the local spin-quench method of Eq. (2) is also shown. It is apparent that the average energy of metastable states is lower for the embedded matching technique, and this behavior is found to survive averaging over the random couplings $\left\{J_{i j}\right\}$. Nevertheless, the probability for converging to a ground state is apparently very small for the system size considered, cf. Fig. 3 . One might speculate that this shortcoming is connected to the fact that only reflections of spins along a plane, i.e., improper rotations, are allowed updates in this approach: if the intermediate, improperly rotated configurations connecting a state to another, properly rotated state of lower energy have higher energy, they form a barrier which cannot be overcome by a strictly downhill procedure. The only other transformation $\mathcal{R}$ besides plane reflections allowing for an Ising type symmetry $\mathcal{R}^{2}=$ id are point inflections $\boldsymbol{S}_{i} \mapsto-\boldsymbol{S}_{i}$. The embedded matching technique can be extended to include these transformations. Their inclusion, however, is not found to remove significantly many barriers, such that this approach is not further considered here [80]. 


\section{BOND-ENERGY DIFFERENCE CROSSOVER AND GENETIC MATCHING}

Although an important improvement over the local spin quench approach (2), embedded matching alone is not sufficient for reliably finding ground states. Further advances are possible by an understanding of the structure of metastable states exploited in a suitably tailored genetic algorithm.

\section{A. Rigidity and domain structure}

To understand the mechanism of metastability in the embedded matching approach and develop a strategy for overcoming it, one needs to take into account some features of the low-temperature phase of spin glasses. While there is no long-range order, the freezing of spin orientations corresponds to some short-range order, expressed in a non-zero range of correlations [2]. Consequently, at low temperatures spins are rather rigidly locked together locally, and their orientation can only be changed (at low, but generally non-zero energies) by a rigid $\mathrm{O}(n)$ rotation of a cluster of spins [48]. Therefore, the manifold of internal states (i.e., the parameter space of the relevant order parameter) is described by the full orthogonal group $\mathrm{O}(n)$, in contrast to the case of homogeneous magnets, where the global magnetization confines the internal states to the quotient space $\mathrm{SO}(n) / \mathrm{SO}(n-1) \simeq S^{n}$, i.e., an $n$-dimensional unit sphere [49, 50]. Such spin clusters hence behave like solid $n$-dimensional bodies. Note, however, that their $\mathrm{O}(n)$ rotation is not in general a zero mode, but a low-energy excitation. The existence of such clusters could recently be explicitly revealed utilizing the genetic embedded matching approach for the planar spin glass in two dimensions [39, 40]. This symmetry also determines the topologically stable defects in spin glasses: as in ferromagnets, they are determined by the homotopy groups of the internal space, here $\mathrm{O}(n)$. For planar rotators, for instance, in addition to vortices (resp. vortex lines) also present in the homogeneous case, this framework predicts domain walls, which can be directly observed in form of chiral walls for the (twodimensional) $X Y$ spin glass [40, 51]. Consequently, some important classes of low-energy excitations in continuous spin glasses are:

1. Rigid $\mathrm{O}(n)$ rotations of spin domains.

2. Topological defects: domain walls, vortices etc.

3. Smooth, spin-wave excitations.

In the context of ground-state searches, spin waves can be easily removed by local relaxation techniques (see the discussion below in Sec IIIB). Some of the topological defects, such as domain walls, can be composed out of a sequence of domain rotations, such that I concentrate on this first type of excitation here. Note that the given classification is not meant to be exhaustive, i.e., it does not (a)

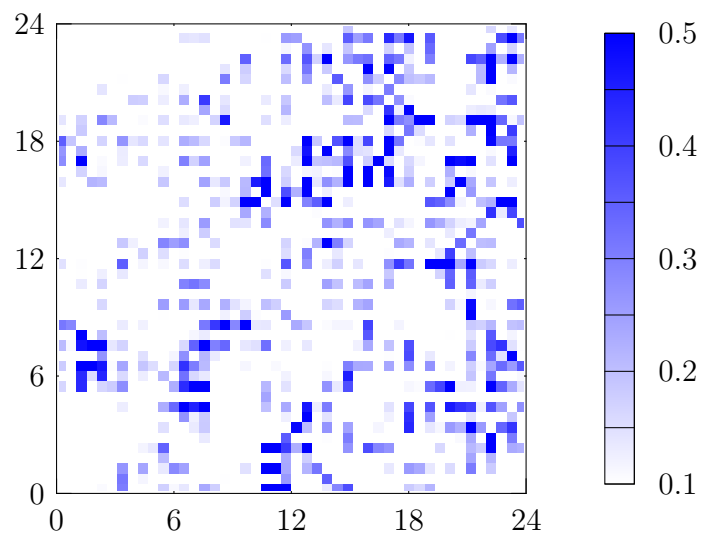

(b)

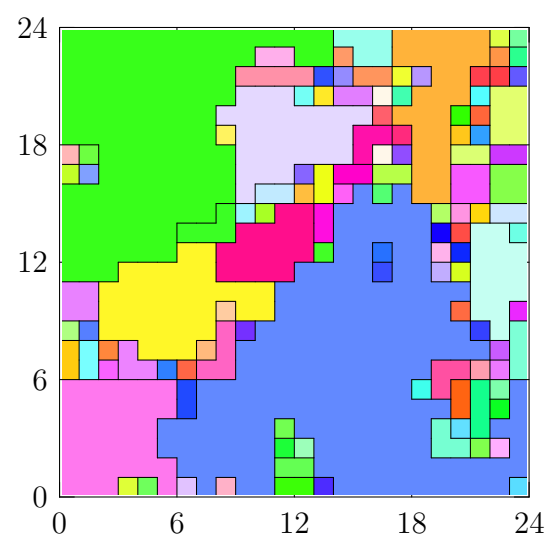

FIG. 4: (Color online) Relative domain decomposition of metastable states of the two-dimensional $X Y$ spin glass. (a) Density plot of bond energy differences (8) for two metastable states of the embedded matching method for a given $24 \times 24$ disorder sample. (b) Cluster decomposition resulting from the Hoshen-Kopelman algorithm with clustering rule (9) and cutoff parameter $\kappa^{\alpha, \beta}=0.3 \sigma_{\mathrm{BED}}^{\alpha, \beta} \approx 0.05$.

express a prejudice as to whether the asymptotic lowenergy excitations in spin glasses are droplets [52, 53, , mean-field like extended defects [54] or "sponges" [55], for instance. It is, instead, only used as a guideline for the identification of appropriate metavariables in the formulation of an efficient ground-state search heuristic.

Given that typical metastable states differ by rigid $\mathrm{O}(n)$ rotations of domains, an explicit implementation of such rotations in an optimization heuristic enables it to perform a search directly on the space of metastable states. Direct inspection of the transformations connecting metastable states in continuous-spin glasses show that this domain structure indeed is a valid description, see Refs. [40, 51, 56]. Note that the concept of such domains is a relative one, i.e., the domain decomposition of a metastable configuration can only be determined with respect to other metastable states. In particular, a domain decomposition may be performed for a pair of configurations, identifying the set of $\mathrm{O}(n)$ rotations map- 
ping them onto each other. This might be achieved by determining, by a singular-value decomposition, locally averaged rotation matrices connecting the configurations [40, 56]. Here, a (computationally) simpler approach is chosen by noting that the $\mathrm{O}(n)$ symmetry of the Hamiltonian (11) ensures that for each such domain all energies $E_{i j}=-J_{i j} \boldsymbol{S}_{i} \cdot \boldsymbol{S}_{j}$ of bonds in the interior are invariant, whereas the energies carried by bonds crossing the domain boundary change due to local mismatches of the surface spins of the rotated domain and its environment. Some of these changes will be of spin-wave type and hence will have been relaxed away once metastability has been reached again. Some differences, however, remain, giving a handle on domain identification. Consequently, for a pair $(\alpha, \beta)$ of metastable configurations one might consider the bond energy differences (BEDs)

$$
\left|\Delta E_{i j}^{\alpha, \beta}\right|=\left|J_{i j}\left(\boldsymbol{S}_{i}^{\alpha} \cdot \boldsymbol{S}_{j}^{\alpha}-\boldsymbol{S}_{i}^{\beta} \cdot \boldsymbol{S}_{j}^{\beta}\right)\right| .
$$

The distribution of BEDs is depicted for a pair of metastable states of the 2D $X Y$ spin glass in Fig. 4(a), showing clear structures of rigid domains. Defining a domain boundary by a BED exceeding a threshold value, i.e.,

$$
\left|\Delta E_{i j}^{\alpha, \beta}\right|>\kappa^{\alpha, \beta},
$$

a domain decomposition can be performed, for instance, with the Hoshen-Kopelman algorithm [57]. This is illustrated in Fig. 4(b) for the BEDs of Fig. 4(a). The cutoff parameter $\kappa^{\alpha, \beta}$ is chosen here of the order of the total variation of BEDs over the whole sample, i.e., proportional to the standard deviation $\sigma_{\mathrm{BED}}^{\alpha, \beta}$, to accommodate differences between disorder realizations as well as metastable states of largely varying energies. Since these domains are merely utilized for a more efficient groundstate search, I do not have to bother here with the question of whether there is a precisely defined characteristic length associated with such domains [56], independent of the size of the system, as having $\kappa^{\alpha, \beta} \sim \sigma_{\mathrm{BED}}^{\alpha, \beta}$ detects the length(s) appropriate to the sample at hand automatically.

\section{B. Genetic matching}

Embedded matching in combination with domain decomposition of configurations with BED clustering allows optimization directly on the space of metastable states of the embedded matching method. This already corresponds to an enormous reduction in the size of the phase space. The meta-optimization on metastable states is performed here utilizing a hybrid genetic algorithm, although variants based on other global optimization strategies such as simulated annealing are conceivable as well. Genetic algorithms [58] mimic natural evolution by maintaining a population of candidate solutions, which is evolved in generations by a process involving the crossover and mutation of solutions followed by a selection of members with higher fitness, i.e., lower energy for the case of ground-state computations considered here. In the canonical form of genetic algorithm, solutions are represented by bit strings in a binary representation and crossover and mutation correspond to the random exchange of bits between solutions and random bit flips, respectively [58]. In this form, genetic algorithms have been applied to the Ising spin glass, but, unless for very small systems, true ground states could not be found with high reliability [59, 60]. Only hybrids combining genetic crossover with some downhill optimization procedure such as local spin flips or the "cluster-exact approximation" [19], restricting the search space to metastable states, led to more successful approaches [20, 61].

Although widely and successfully employed, there is no theoretically sound framework for designing efficient genetic algorithms [58], such that their construction rests on heuristic strategies and additional insight specific to the problem at hand. Generally, one strives to achieve a balance between fast convergence to an optimum answer and the upholding of genetic diversity throughout the "evolution" (which, in turn, tends to slow down convergence), in order not to miss the global optimum. The choice of crossover operation appears to be most important in this context. In the present work, instead of randomly exchanging single spins, the BED cluster decomposition is employed to exchange domains of rigid spins between solutions. This has the important advantage of retaining the high degree of optimization already found from the embedded matching technique inside of the domains and directly operating on the space of variables relevant for the construction of metastable states. The domain decomposition can be performed directly with the "parent" configurations to be combined ("diadic" crossover) or, alternatively, by using a third, "mask" configuration from the population used only for the domain decomposition. The latter, "triadic" crossover is used here, similar to the technique suggested in Ref. 62] for Ising spins, since it is found to perform slightly better for continuous spins. Genetic diversity is strengthened by restricting the selection of parents to be combined to neighbors after the population has been arranged in a linear ring 62]. This introduces some degree of geometric "locality" of the population and allows good solutions to be refined independently in different areas of the configuration set. Previous approaches [20, 61, 63] have used a fixed total number of crossover operations per member of the initial population, followed by a halving of the population by elimination of the higher-energy solution of each pair of neighboring configurations, and then a reiteration of the remaining population. This reduces the total effort by removing unpromising solutions and bringing distant parts of the "ring" of solutions closer to each other in later stages of the optimization. For the time being, I will adopt this technique here as well. A more efficient variant, geared at the detection of hard samples, is presented below in Sec. IVC In total, the resulting 
genetic embedded matching (GEM) algorithm proceeds as follows:

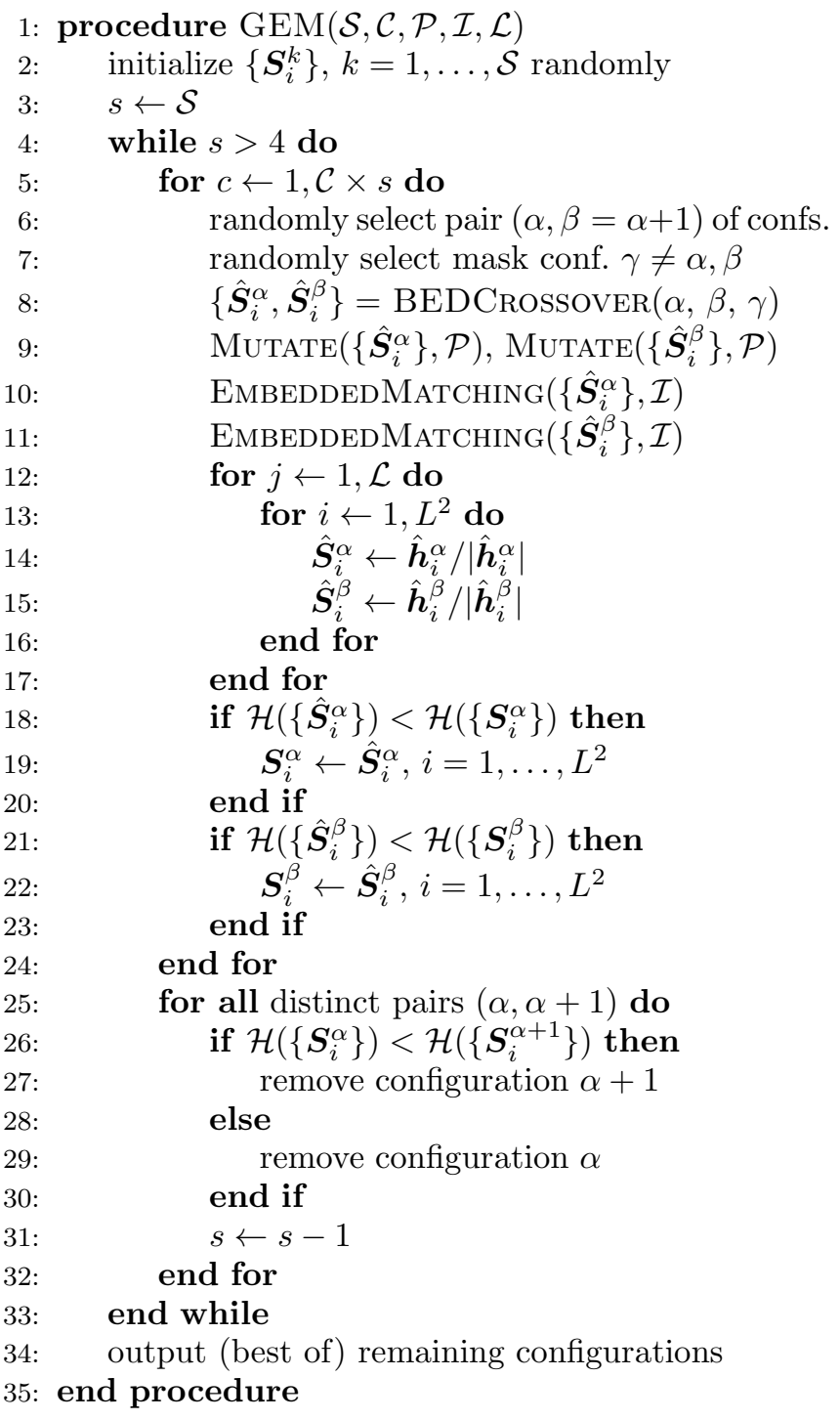

The BEDCRossover operation performs the BED domain decomposition of Sec. IIIA with respect to the mask and, for each domain, either swaps all spins between the parents $\alpha$ and $\beta$, copies domains $\alpha \mapsto \beta$ or $\beta \mapsto \alpha$, or leaves the domain invariant, with all possibilities occurring randomly at equal proportions. Mutations are performed by randomly choosing new spin orientations with a probability $\mathcal{P}$. The resulting offspring are optimized using $\mathcal{I}$ iterations of EMBEDDEDMATCHING from Sec. ПB followed by $\mathcal{L}$ iterations of a local spin quench. The latter is useful since, close to a minimum where only spin-wave excitations are left, both approaches converge to the same state, but the spin quench is much faster computationally. Lower-energy offspring then replace their parents. In the implementation used, each offspring is only compared to the morphologically closer parent, i.e., the one with a larger optimized scalar overlap $\hat{q}^{\alpha \beta}=\max _{R \in \mathrm{O}(n)} \sum_{\sigma, \tau} q_{\sigma \tau}^{\alpha \beta} R_{\sigma \tau}^{\alpha \beta}$, where $R_{\sigma \tau}^{\alpha \beta}$ de- notes the corresponding global rotation matrix, and

$$
q_{\sigma \tau}^{\alpha \beta}=\frac{1}{L^{2}} \sum_{i} S_{i \sigma}^{\alpha} S_{i \tau}^{\beta}
$$

is the matrix of overlaps. This maximization is performed by a singular value decomposition to diagonalize $q_{\sigma \tau}^{\alpha \beta}$, in which case $\hat{q}^{\alpha \beta}$ is just the trace of the resulting diagonal $q_{\sigma \tau}^{\alpha \beta}[40,56]$. This form of replacement restriction helps to maximize genetic diversity [20]. After $s \mathcal{C}$ crossovers, the higher energy instance of each adjacent pair of configurations is discarded, thus halving the population. The complete process is repeated until at most four configurations are left, which form the result of a run.

As will be shown in the next Section, this combination of techniques in the genetic embedded matching method allows for the determination of (numerically) exact ground-states of reasonably large continuous-spin systems in $2 \mathrm{D}$ with high reliability.

\section{PERFORMANCE}

Using probabilistic methods for ground-state searches, special care is needed to ensure that true ground states are found. Since for $\mathcal{N P}$ hard optimization problems the decision variant is $\mathcal{N P}$ complete, there is no way of definitely distinguishing a metastable from a ground state short of an exact solution of the instance. A general probabilistic approach of "quality assurance" for the GEM method is outlined and applied to the 2D $X Y$ spin glass in Sec. IVB In some dynamical approaches, such as local spin-flip Monte Carlo simulations, the specific hardness of a sample shows up in the behavior of autocorrelation times, to which a simulation run can in principle react dynamically by increasing the simulation time accordingly. Below in Sec. IVC it is discussed whether a similar heuristic for detecting hard samples can be applied in the GEM approach.

\section{A. Performance and comparison to simulated annealing}

Local spin quenches according to Eq. (2) yield states in a broad range of energies, cf. Fig. 3. For ascribing the ability to find ground states to a stochastic method one would, instead, require that states of exactly the same energy (up to machine precision) are found in a sizable fraction $p_{s}$ of attempts (with $p_{s}=95 \%$, for instance) and that no states of lower energy can be found with runs of largely increased effort or utilizing other optimization techniques. As is evident from Fig. 3, this also cannot be said of the embedded matching approach alone. Figure 5 shows the minimum energies found in repeated runs of the GEM technique for the bimodal $X Y$ spin glass in $2 \mathrm{D}$ with a randomly picked disorder realization of size $24 \times 24$ (which is identical to the realization used in Fig. (3) 


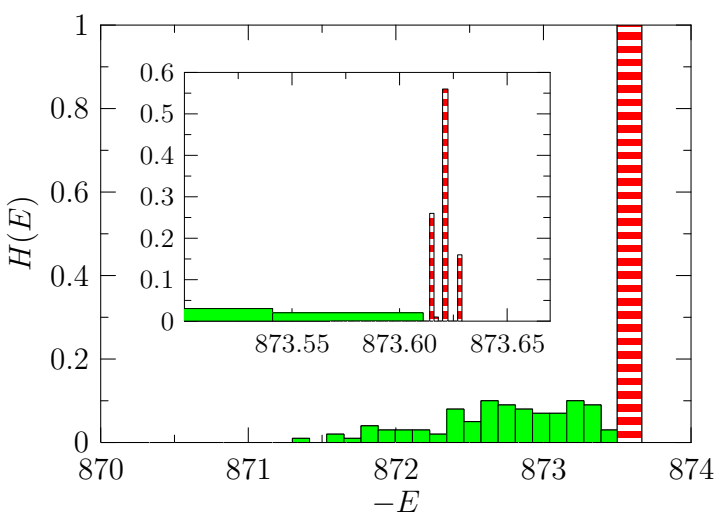

FIG. 5: (Color online) Histograms of energies found for a $24 \times 24$ sample of the bimodal $X Y$ spin glass from 100 runs of the genetic embedded matching approach with a population of size $\mathcal{S}=64$ (hatched bars, average runtime $700 \mathrm{~s}$ on a Pentium IV $2.8 \mathrm{GHz}$ ) as compared to simulated annealing runs with a total of about $2 \times 10^{7}$ Monte Carlo sweeps (solid bars, average runtime $4000 \mathrm{~s}$ ). The disorder realization is identical to the one considered in Fig. 3

and a starting population size $\mathcal{S}=64$. For comparison, this Figure also shows the histogram of repeated runs of an extensive simulated annealing [16, 64] computation with an exponential temperature protocol (a linear protocol yields very similar results) and a total number of about $2 \times 10^{7}$ lattice sweeps of single spin flips per run, leading to an about sixfold runtime as compared to the GEM computations. As is seen, the GEM runs result in clearly lower energies than the simulated annealing. Additionally, the latter still show a sizable spread of the energies found, whereas the GEM technique appears to yield states of the same energy. Only on going to much higher energy resolution, the GEM results are resolved into a small number of distinct peaks cumulated from runs yielding identical energy up to (or close to) machine precision, cf. the inset of Fig. 5 . A fourfold increase of the starting population to $\mathcal{S}=256$ leads to a convergence of all 100 runs to the lowest-energy peak to the right in the inset of Fig. 5. No further increases of the population size up to $\mathcal{S}=1024$ lead to lower energies such that, with high confidence, this peak corresponds to the true ground-state energy of the system. Consequently, it can be said that runs with $\mathcal{S}=64$ have a probability of about $p_{s}=16 \%$ of leading to a ground-state. Methods for guaranteeing high reliability of finding ground states over the distribution of disorder realizations are discussed below in Secs. IVB and IVC,

Although, for the given disorder realization, the GEM technique appears able to find ground states and clearly outperforms the simulated annealing approach, variations in the "hardness" of different replica in the random couplings are known to be large (see, e.g., Refs. 65, 66]), and the corresponding variation in the efficiency of the methods should be taken into account. Since the com-

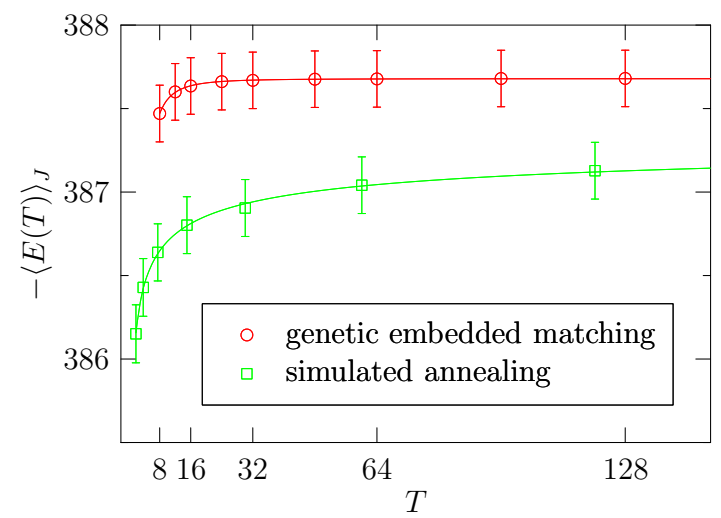

FIG. 6: (Color online) Average minimum energy $\langle E(T)\rangle_{J}$ for 1000 samples of size $16 \times 16$ of the bimodal $2 \mathrm{D} X Y$ spin glass found from GEM and simulated annealing runs with a total runtime $T$ (in re-scaled units).

monly considered distributions $P\left(J_{i j}\right)$ contain the ferromagnetic lattice with $J_{i j}=J_{0}>0$, which is trivially handled by either optimization method, the behavior of interest can only be either that for the worst case, which is, however, difficult to assess for the spin-glass model considered, or rather the average performance for the disorder distribution at hand. As a first step in this analysis, I considered the convergence of the average minimum energy observed with the computational effort invested. For simulated annealing with Metropolis acceptance rule, it is known that with logarithmically slow cooling, ground states will be found in finite (but, of course, very large) time 67]. Since this cooling schedule is impractical, however, exponential or power-law cooling curves are used instead [16, 64]. The possibility of different acceptance rules complicates things further, and it is naturally impossible to benchmark against all these variants. I restrict myself here to the probably most commonly used exponential protocol. The asymptotic form of energy convergence in simulated annealing of spin-glass systems has been the topic of some debate in the past [5, 25]. Numerically, a power-law convergence,

$$
\langle E(T)\rangle_{J} \sim E_{\infty}+A_{E} T^{-\zeta},
$$

for large cooling times $T$ (i.e., the total number of Monte Carlo sweeps) was found for the 2D Ising spin glass, while, on the contrary, logarithmic convergence was observed for the $3 \mathrm{D}$ variant [25]. On the other hand, on the basis of modeling (Ising) spin glasses as sets of weakly interacting two-level systems, it was conjectured that the logarithmic form would be universal $[5]$. Here, $\langle\cdot\rangle_{J}$ denotes the average over disorder. Figure 6 shows the average minimum energy found from simulated annealing of 1000 samples of size $16 \times 16$ of the $2 \mathrm{D} X Y$ spin glass for annealing times between $T=50000$ and $T=3.2 \times 10^{6}$ sweeps, compared to the energies found from GEM runs with population sizes $\mathcal{S} \propto T$ between 8 and 128 configurations. The abscissa for the simulated annealing data has 
been linearly rescaled to result in equal runtimes for both approaches on a Pentium IV $2.8 \mathrm{GHz}$ (both algorithms scale linearly in $T$ ). The data from simulated annealing can be fitted with reasonable quality to the powerlaw form (11), yielding a decay exponent $\zeta=0.33(36)$, whereas a logarithmic form does not adequately describe the data. This is comparable to the value $\zeta=0.25$ found for the 2D Ising spin glass in Ref. 25]. Note that the extrapolated asymptotic ground-state energy $E_{\infty}=-387.45(76)$ is compatible statistically with the value found from GEM runs already for the smallest population size $\mathcal{S}=8$ considered. In fact, the GEM data are constant within statistical errors for $\mathcal{S} \geq 32$. The variation of energies found from the GEM technique can also be described by (11), resulting in $\zeta=2.3(48)$ (the large statistical error results from the only minute variation of $\langle E\rangle$ observed).

The GEM algorithm as presented in Sec. IIIB involves a number of parameters which need to be tuned to achieve these good results. Performance appears to be rather weakly dependent on the mutation rate, and best results are found for a rate of about $\mathcal{P}=2.5 \%$. Much more frequent mutation destroys the relatively good optimization achieved at intermediate stages and decrease the overall performance. Since the offspring configurations produced by the BED crossover are still optimal inside of domains, relatively small numbers of embedded matching and local relaxation steps are found to be sufficient, $\mathcal{I}=15$ and $\mathcal{L}=100$ was usually chosen here (cf. the pseudocode of the algorithm in Sec. IIIB). The number $\mathcal{C}$ of crossovers per replica determines how well the available "genetic pool" of original configurations is explored. Beyond a certain number of crossovers, the population becomes uniform and further increases do not improve the probability of finding ground states. For accessible system sizes $\mathcal{C}=8-16$ is a good choice. The main tuning parameter of the approach is the initial population size $\mathcal{S}$, which is changed to accommodate the variable hardness of different system sizes, models and disorder realizations. It is the only parameter whose increase ultimately guarantees ground states to be found. Note that the total number of crossovers is $2 \mathcal{C}(\mathcal{S}-4)$ (assuming $\mathcal{S}=2^{n}$ ) and hence linear in $\mathcal{S}$. For a given single realization, computation of true ground states can be guaranteed with high confidence by tackling the same disorder configuration with largely increased computational effort (in particular, say, a fourfold increase in population size $\mathcal{S}$ ), until no further change in energy is observed. For the random distribution of configurations to be investigated, however, a more automatic (and less computationally expensive) approach is required.

\section{B. Probabilistics of successes}

For stochastic optimization methods, arrival at true ground states cannot be guaranteed. For given input data in form of the disorder realization and a choice for

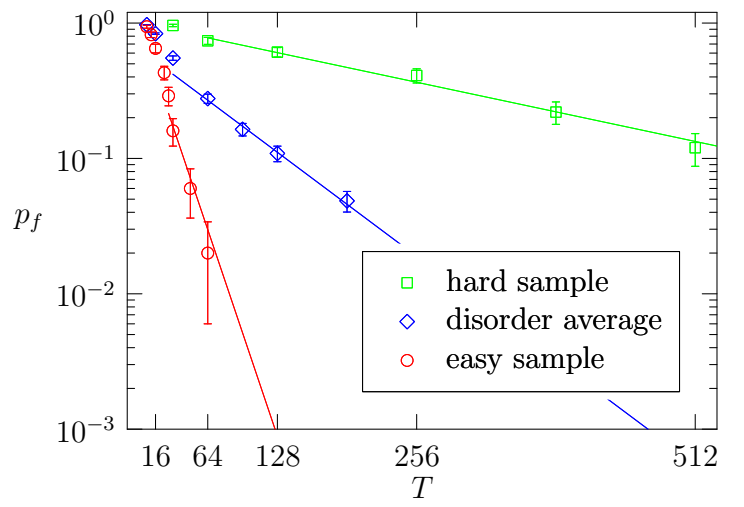

FIG. 7: (Color online) Estimated failure probability $p_{f}=1-$ $p_{s}$ for the GEM technique applied to $X Y$ spin-glass samples of size $16 \times 16$ as a function of the computational effort $T=\mathcal{S}$. The lines show fits of the form (12) (easy and hard sample) resp. (13) (disorder average) to the data.

the tunable parameters, a ground state is found with the success probability $p_{s}\left(\left\{J_{\mathrm{ij}}\right\} ; T\right)$, where $T$ denotes the relevant parameters. As was discussed above, by far the most influential parameter for the GEM approach is the initial population size $\mathcal{S}$, such that I here restrict considerations to $T=\{\mathcal{S}\}$. Full information about the distribution of $p_{s}$ induced by $P\left(\left\{J_{i j}\right\}\right)$ and the dependency on the algorithm's parameters would correspond to a complete understanding of the performance characteristics or generalized computational complexity [7]. This computation, however, is impractical due to the high-dimensional nature of this parameter space: using, e.g., 100 runs to estimate $p_{s}$ for a given set of parameters for 1000 disorder realizations and 100 combinations of parameters $\mathcal{S}, \mathcal{I}, \ldots$ would require $10^{7}$ ground-state computations for a single system size! From $p_{s}\left(\left\{J_{\mathrm{ij}}\right\} ; T\right)$ one could deduce the perhaps most interesting distribution $T_{\min }\left(\left\{J_{\mathrm{ij}}\right\} ; p_{s}\right)$ of efforts required for a constant success probability $p_{s}$.

Figure 7 shows the failure probabilities $p_{f}=1-p_{s}$ for an "easy" and a "hard" sample of the $2 \mathrm{D}$ bimodal $X Y$ spin glass as a function of the population size $\mathcal{S}=T$, compared to the average failure rate $p_{f}$ over 100 disorder replica. The huge spread in hardness is apparent: while, for instance, only in 2 out of 100 cases do runs with $\mathcal{S}=64$ fail to find a ground state for the easy sample, $74 \%$ of attempts for the hard sample end in a metastable state. It is therefore not enough to fix the run parameters by considering one or two randomly chosen configurations. For a description of the functional form of $p_{f}(T)$ in Fig. 7 consider performing $n$ statistically independent runs of length $T_{0}$ with failure probability $p_{f, 0}$ and picking the solution of lowest energy as final answer. With this prescription, a ground state is not being found only if all of the runs fail, and the combined failure probability is thus

$$
p_{f}\left(T=n T_{0}\right)=p_{f, 0}^{T / T_{0}}
$$




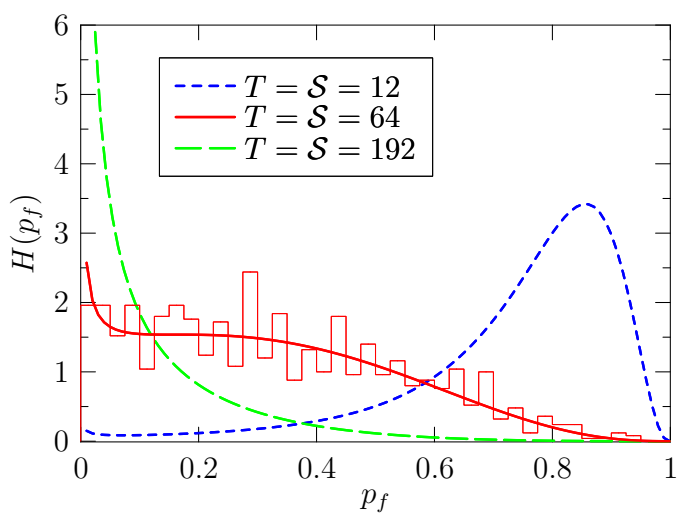

FIG. 8: (Color online) Histogram $H\left(p_{f}\right)$ of failure probabilities for 1000 disorder samples for the 2D $X Y$ spin glass as a function of initial population size $\mathcal{S}$. The lines show analytical approximations discussed below in the main text.

Due to the locality constraint in choosing parent configurations for crossover, increasing the initial population size $\mathcal{S}=T$ has essentially the same effect as performing independent runs. For sufficiently large $T$, Eq. (12) is hence an excellent description of $p_{f}(T)$ for a single sample. Figure 7 shows fits of the form (12) to the data for the "easy" and "hard" samples. There is only a single fit parameter, $p_{f, 0}^{1 / T_{0}}$, corresponding to a measure of hardness of the sample (with respect to the GEM technique). The ensemble average $\left\langle p_{f}\left(\left\{J_{i j}\right\} ; T\right)\right\rangle_{J}$ cannot be expected to follow the same exponential form (12) since, in general, $\langle\exp [\alpha T]\rangle \neq \exp [\langle\alpha\rangle T]$. It is found, however, that it is well described by the slight generalization

$$
\left\langle p_{f}\left(\left\{J_{i j}\right\} ; T\right)\right\rangle_{J}=A_{p} p_{f, 0}^{T / T_{0}},
$$

with an additional amplitude $A_{p}<1$, as is apparent from the corresponding fit also shown in Fig. 7 Consequently, the average failure probability decreases more slowly with $T$ than would be expected from the behavior on single samples. Note that due to the form (12) it is not appropriate to consider the combination $T / p_{s}$ as the "computational effort" of a sample [66], since this assumes a linear relation between $p_{s}$ and $T$.

In view of the results of Fig. 7 it is of interest to investigate the distribution $H\left(p_{f}\right)$ of failure probabilities over disorder samples. To this end, the failure probability was sampled by performing 100 independent runs for each of 1000 disorder samples with run length $T=\mathcal{S}=64$. The histogram estimating the probability-density function $H\left(p_{f}\right)$ is shown in Fig. 8, revealing clearly the breadth of this distribution, reflecting the large spread in hardness already suggested by the data of Fig. 7. From the histogram $H\left(p_{f}\right)$, it is possible by means of Eq. (12) to recover the distribution of the minimum required runtimes $T_{\min }\left(\left\{J_{\mathrm{ij}}\right\} ; p_{f}\right)$, corresponding to the distribution of hardness of samples under the GEM technique: from the estimate of $p_{f}\left(\left\{J_{i j}\right\}\right)$ for a disorder sample $\left\{J_{i j}\right\}$ at

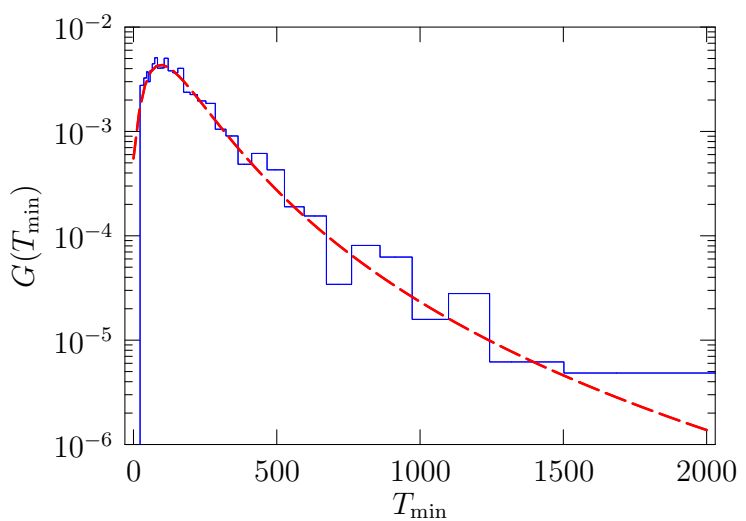

FIG. 9: (Color online) Estimated probability-density function $G\left(T_{\min }\right)$ of minimum required runtimes $T_{\min }$ for $16 \times 16$ systems of the $2 \mathrm{D} X Y$ spin glass to achieve success probability $p_{s}=95 \%$. The dashed line shows a fit of the generalized extreme-value distribution (15) to the data.

fixed runtime $T$, Eq. (12) implies

$$
T_{\min }\left(\left\{J_{\mathrm{ij}}\right\}\right)=T \frac{\ln p_{f, 0}}{\ln p_{f}\left(\left\{J_{i j}\right\}\right)} .
$$

Figure 9 shows the distribution $G\left(T_{\min }\right)$ of minimum runtimes at failure probability $p_{f, 0}=0.05$ thus resulting from the runs at fixed runtime $T=64$ presented in Fig. 8. The breadth of the distribution is apparent: while the average is around $\left\langle T_{\min }\right\rangle_{J} \approx 225$, there is a fat tail with some of the 1000 disorder configurations featuring a $T_{\min }$ as large as 2000 . Such density functions typically occur when considering the extrema of large samples drawn from underlying probability distributions. Asymptotically, the distribution of extremes is known to be universal, following the form [68]

$$
\begin{aligned}
G_{\xi, \mu, \sigma}(x)= & \frac{1}{\sigma}\left(1+\xi \frac{x-\mu}{\sigma}\right)^{-1-1 / \xi} \\
& \times \exp \left[-\left(1+\xi \frac{x-\mu}{\sigma}\right)^{-1 / \xi}\right],
\end{aligned}
$$

where the parameter $\xi$ depends on the tail behavior of the underlying, primary distribution for large arguments $x$. Depending on $\xi$, this form is known as Weibull $(\xi<0)$, Gumbel $(\xi \rightarrow 0)$ or Fréchet $(\xi>0)$ distribution, respectively. As is seen in Fig. 9, it fits the data for $T_{\min }$ extremely well, resulting in $\xi=0.270(44)$, $\mu=115.6(45)$, and $\sigma=87.7(39)$ with an excellent quality-of-fit $Q=0.23$. Similar distributions of the Fréchet type have been found for the tunneling times in Wang-Landau flat-histogram simulations of the Ising spin glass [66, 69]. One might speculate on the origin of the occurrence of extreme-value statistics in hardness measures of spin-glass samples: if, as has been suggested [5], a spin-glass sample can be described as a set of $n=n(L)$ weakly interacting two-level systems, it appears plausible that the largest barrier or the slowest relaxation time 
determines the hardness of the configuration. Then, the hardness would be the maximum or minimum of a sample of size $n$ from the underlying distribution of twolevel systems, asymptotically distributed according to the extreme-value distribution (15). In line with this argument, it was recently observed [70] that the distribution of relevant barriers in the Sherrington-Kirkpatrick model follows a Fréchet distribution with a value of $\xi \approx 0.33$, rather similar to the form found here.

Given that $G_{\xi, \mu, \sigma}\left(T_{\min }\right)$ describes the GEM data so well, it is worthwhile to use Eq. (12) to reveal the resulting analytical form of the distribution of failure probabilities, $H\left(p_{f}\right)$. Following standard probability theory [71], density functions transform as

$$
H\left(p_{f}\right) \mathrm{d} p_{f}=G_{\xi, \mu, \sigma}\left(T_{\min }\right) \frac{\mathrm{d} T_{\min }}{\mathrm{d} p_{f}} \mathrm{~d} p_{f},
$$

which, using Eq. (12), leads to

$$
H\left(p_{f}\right)=-\frac{T \ln p_{f, 0}}{p_{f}\left(\ln p_{f}\right)^{2}} G_{\xi, \mu, \sigma}\left(T \frac{\ln p_{f, 0}}{\ln p_{f}}\right) .
$$

As is seen from the curves in Fig. 8 this form with the parameter values $\xi, \mu$ and $\sigma$ given above fits the numerical distribution $H\left(p_{f}\right)$ for the same $T=64$ data perfectly well (i.e., the approach is self-consistent). Additionally, however, it describes independent runs of different lengths to high precision and hence the form (17) is an excellent description for general runtimes $T$, as indicated by the additional curves in Fig. 8 . Consequently, the three-parameter family of distributions (15) and the limiting distributions derived via Eq. (12) form a complete description of the full probability density $p_{f}\left(\left\{J_{\mathrm{ij}}\right\} ; T\right)$.

While it certainly would be instructive to extend the analysis of $T_{\min }$ via the distributions (15) to a scaling analysis of the fit parameters $\xi, \mu$ and $\sigma$ with lattice size $L$, the huge computational effort would be disproportional. Instead, I concentrate on the mean required effort $\left\langle T_{\min }\right\rangle_{J}$ as a function of system size, averaged over a smaller disorder sample of only 100 configurations. These data for failure probability $p_{f}=5 \%$ are shown in Fig. 10 together with a fit to the expected exponential form

$$
\left\langle T_{\min }\right\rangle_{J}=A_{T} e^{\left(L / L_{0}\right)^{2}},
$$

which works reasonably well with parameters $A_{T}=$ $4.82(26)$ and $L_{0}=8.515(89)$. Increasing the rate of tolerated failures to, e.g., $p_{f}=10 \%$, merely reduces the prefactor to $A_{T}=3.64(19)$, but leaves $L_{0}$ almost invariant. This data brings back to attention the fact that, although the GEM approach works quite well, and clearly outperforms simulated annealing, it naturally cannot evade the $\mathcal{N} \mathcal{P}$-hard nature of the problem enforcing an exponential growth of computational effort. To complicate the matter further, it is well conceivable that the shape parameter $\xi$ of (15) increases with system size, as was observed in tunneling simulations of spin-glass models [66]. Since for $\xi>1 / 2$ the variance of $G_{\xi, \mu, \sigma}$ becomes ill-defined, and

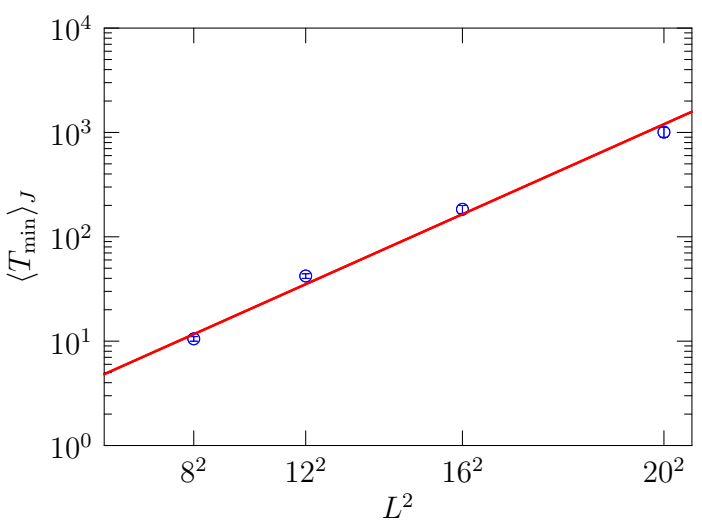

FIG. 10: (Color online) Average effort $\left\langle T_{\min }\right\rangle_{J}$ for finding ground states with constant success probability $p_{s}=95 \%$ as a function of lattice size $L^{2}$, estimated from 100 disorder configurations per size. The line shows a fit of the form (18) to the data.

for $\xi>1$ additionally the mean diverges, this would imply that a correct choice of population size $\mathcal{S}=T$ for all disorder configurations becomes impossible beyond a certain system size.

\section{Hardness of samples and refinements}

Numerical ground-state computations in (nonpolynomial) spin-glass systems are subject to two types of "hardness problems": the exponential growth of average computational effort with system size and the large fluctuations in sample hardness implied by heavy-tailed distributions of the type (15). While $\mathcal{N} \mathcal{P}$-hardness means exponential effort for the worst-case samples, it is clear that (close to) ferromagnetic (i.e., best case) configurations can be tackled in polynomial time. Hence, the difference in effort diverges with system size. While this is true for the set of all possible input data, it is not clear a priori that the samples receiving non-negligible weight from the $P\left(\left\{J_{i j}\right\}\right)$ considered indeed show such spread as implied by the data of Fig. 9. In this Section, I discuss technical refinements of the GEM technique designed to address the problem of large fluctuations in hardness.

\section{Effort adaptation}

The fixed total number of crossovers $C_{\text {tot }}=2 \mathcal{C}(\mathcal{S}-4)$ performed by the GEM algorithm of Sec. IIIB is not optimal in view of the hardness variations observed. Additionally, one needs to tune $\mathcal{C}$ for best performance. It turns out that, in fact, the number of crossovers can be determined automatically and on the run. This is done by comparing each pair of configurations generated by crossover and potential replacement of the parents: if 


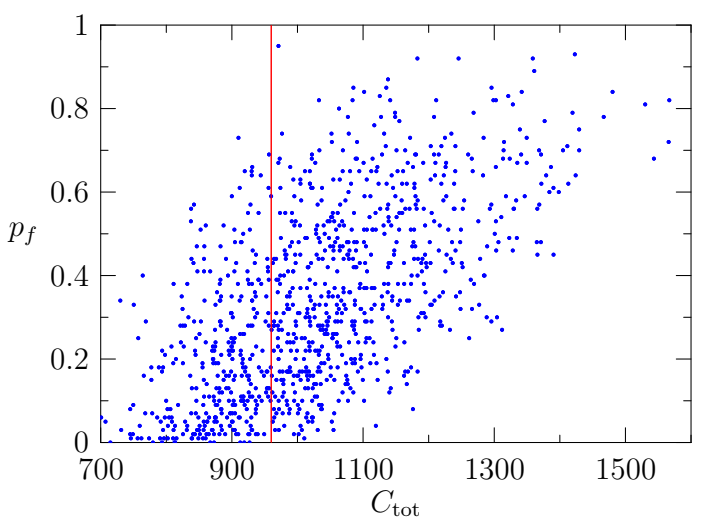

FIG. 11: (Color online) Correlation diagram of failure probabilities $p_{f}$ for GEM runs of length $\mathcal{S}=64$ and the total number of crossovers $C_{\text {tot }}$ in ODGEM (overlap-driven GEM) runs with $\mathcal{D}=0.128$ for $16 \times 16$ disorder samples. The vertical line shows the total number $C_{\text {tot }}=960$ of crossovers for the GEM runs.

they are too similar, one of them is removed from the population. The similarity is here again measured by the optimized scalar overlap $\hat{q}^{\alpha \beta}$ resulting from Eq. (10), using a cutoff $q_{\max }=1-2 \mathcal{D} / L^{2}$. For an Ising spin glass, $\mathcal{D}$ would correspond to the number of lattice sites where the two configurations disagree. For the algorithm of Sec. IIIB this means that the loop over $c$ in lines 5 and 24 as well as the halving step in lines 25-32 are removed, whereas the following instructions are inserted after line 23:

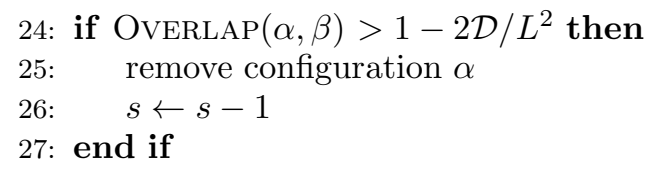

This modified algorithm is referred to here as ODGEM (overlap-driven GEM). This prescription ensures maximal genetic diversity at all times, and only members able to produce "novel" configurations in crossover are retained in the population. As a consequence, the total number of crossovers is no longer fixed, but depends on the used disorder configuration. It appears plausible that hard disorder samples with many conflicting solutions close to the global minimum retain genetic diversity longer than easy samples. Figure 11 shows a correlation plot between the failure probabilities $p_{f}$ in the original GEM and the total number of crossovers $C_{\text {tot }}$ in the ODGEM approach (which is proportional to the total computational effort). The Pearson correlation coefficient [7],

$$
\rho_{X, Y}=\frac{\langle[X-\langle X\rangle][Y-\langle Y\rangle]\rangle}{\sigma_{X} \sigma_{Y}}
$$

is found to be $\rho_{p_{f}, C_{\text {tot }}}=0.625(15)$, indicating clear but not perfect correlation. As is seen from Fig. 11, most

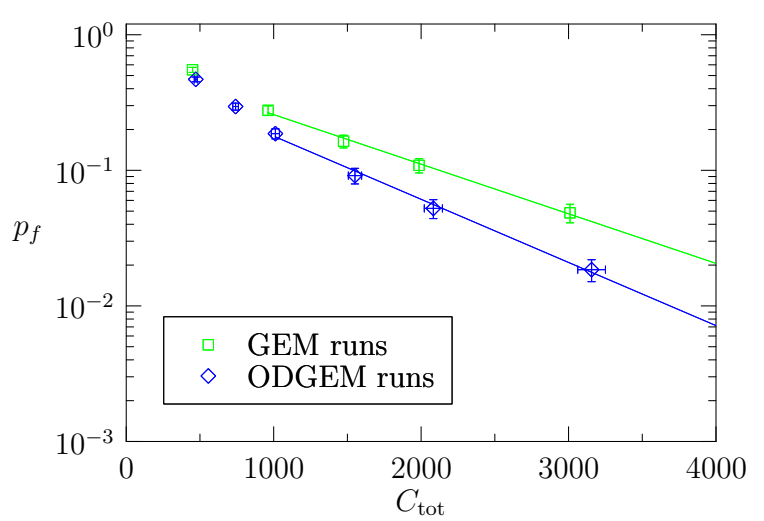

FIG. 12: (Color online) Average failure probabilities for the GEM and ODGEM techniques on $16 \times 16$ samples as a function of the total number $C_{\text {tot }}$ of crossovers performed. The lines show fits of the functional form (13) to the data.

samples with high failure rates in GEM receive an increased effort in ODGEM, and only a few cases are missed. The choice of the overlap cutoff $\mathcal{D}$ must ensure that when comparing configurations genetic "equality" is assumed only when no relative, non-trivial excitation exists. Values of $\mathcal{D}=0.1$ to $\mathcal{D}=1$ are found appropriate here.

Since the ODGEM method detects a significant proportion of hard samples, the distribution of the total number $C_{\text {tot }}$ of crossovers acquires itself a heavy tail, reflecting the hardness distribution described by Eq. (15). This empirical distribution is found to be well modeled by the extreme-value shape (15) with $\xi$ close to zero, i.e., a Gumbel form. As is evident from the data presented in Fig. 12, this leads to an improved average performance of the ODGEM compared to the GEM technique, increasing with the total effort invested. Compared to GEM, ODGEM invests more effort in the hard samples and less in the easy ones, as appears adequate. Direct inspection of the histogram $H\left(p_{f}\right)$ of failure probabilities for the ODGEM method reveals that, indeed, the number of instances with large failure probabilities are dramatically reduced as compared to the GEM data of the same population size presented in Fig. 8

\section{Hardness indicators}

The possibility of deciding about sample hardness $a$ priori could largely increase the efficiency and reliability of the GEM (and any other ground-state search) method. It is an open problem whether for spin-glass systems there are microscopic sample properties significantly more easily computable than the ground state itself which feature a strong correlation to sample hardness (with respect to a given method) [7, 72, 73]. To investigate this question in the context of the GEM approach and the 2D bimodal $X Y$ spin glass, I analyzed corre- 
lations between the failure probabilities $p_{f}$ and a large number of observables of the disorder sample, the candidate ground states found, and properties of the population of configurations in the genetic algorithm. A number of resulting correlation coefficients as estimated for $16 \times 16$ samples from GEM and ODGEM runs with population size $\mathcal{S}=64$ are collected in Tab. I.

Regarding easily computable properties of the disorder realizations at hand, one finds some moderately significant, positive correlation between $p_{f}$ in GEM runs and the number of frustrated plaquettes, indicating increased hardness for highly frustrated samples. Vice versa, an increasing average size of unfrustrated regions leads to more and more success in finding ground states. Apparently, these correlations are successfully taken into account by the modified algorithm ODGEM, where no significant correlations between $p_{f}$ and sample properties are left. Also, some properties of the computed (candidate) ground states correlate with success probabilities. In particular, a larger ground-state energy, indicating increased frustration, is accompanied by a larger number of failures. Also, larger than average values of the configurational r.m.s. chirality $\kappa[74]$,

$$
\kappa^{2} L^{2}=\sum_{\square_{n}}\left\{\sum_{(i, j) \in \square_{n}} \operatorname{sign} J_{i j}\left[\boldsymbol{S}_{i} \times \boldsymbol{S}_{j}\right]_{z}\right\}^{2},
$$

as well as the non-collinearity $Q$ [75],

$$
Q^{4} L^{4} / 2=\sum_{\square_{n}} \sum_{(i, j) \in \square_{n}}\left|\boldsymbol{S}_{i} \times \boldsymbol{S}_{j}\right|^{2},
$$

correlate with larger failure probabilities and hence indicate harder samples for the method. Note that the definition(20) is specific to the case of planar spins. For the Heisenberg model, the chirality is, instead, cubic in the spin variables [76]. Again, such correlations are not (highly) significant for the ODGEM technique, indicating that the corresponding samples automatically receive higher computational effort there.

The largest correlations with the failure probability are seen for properties of the population of spin configurations inside of the GEM or ODGEM runs. The rather strong inverse correlation between the average optimized overlap $\left\langle\hat{q}^{\alpha \beta}\right\rangle$ of configurations and $p_{f}$ in GEM runs shows that a homogeneous (i.e., large overlap) population occurs for a clear-cut, more easily accessible ground state. Such homogeneity also implies that larger domains are being identified in the BED decomposition. On the contrary, a large number of successful replacements of parents by better offspring indicates stronger competition of candidate ground states, resulting in more failures. These correlations related to population remain, although weakened, in the maximum-diversity version ODGEM. Consequently, devoting additional effort to disorder configurations singled out by these population characteristics in ODGEM runs will additionally reduce failure rates for hard samples, as I now discuss.

\begin{tabular}{lrr}
\hline \hline Observable & \multicolumn{1}{c}{$\rho_{\text {GEM }}$} & \multicolumn{1}{c}{$\rho_{\text {ODGEM }}$} \\
\hline No. AF bonds & $0.020(36)$ & $0.019(34)$ \\
No. frustrated plaquettes & $0.104(33)$ & $-0.028(33)$ \\
Size of unfrust. clusters & $-0.129(38)$ & $-0.012(37)$ \\
\hline Energy & $0.226(33)$ & $0.061(34)$ \\
Magnetization & $0.021(34)$ & $0.024(36)$ \\
Chirality & $0.137(34)$ & $0.001(33)$ \\
Non-collinearity & $0.266(33)$ & $0.101(33)$ \\
\hline Plain overlap & $-0.087(31)$ & $0.059(32)$ \\
Optimized overlap & $-0.414(25)$ & $-0.339(28)$ \\
BED domain size & $-0.383(22)$ & $0.237(34)$ \\
Parent replacements & $0.328(29)$ & $0.297(34)$ \\
\hline \hline
\end{tabular}

TABLE I: Estimated correlation coefficients between the failure probabilities $p_{f}$ in GEM and ODGEM runs and various observables of the disorder realizations, the actual groundstate configurations found, and the population of configurations within the genetic algorithm.

\section{Repeated runs}

Allocating such additional effort to allegedly hard samples typically means performing additional, statistically independent runs to finally pick the lowest-energy state found as the final result. As discussed above in Sec. IVB, however, the decrease in failure probability expected from such a combination of several runs as described by Eq. (12) is identical to the effect of performing a single computation with a larger population (and this stays true for the modified ODGEM technique, at least to a good approximation). In contrast to single, more expensive runs for all disorder realizations, however, repeated runs allow to treat individual realizations differently, in accord with the heavy-tailed distribution of sample hardness observed in Fig. 9.

Even disregarding the use of the hardness indicators discussed in the previous Section, however, it turns out to be beneficial to replace runs of length $T$ by a number $n$ of shorter runs of length $T / n$ : within the range of validity of Eq. (12), the total probability $p_{f}$ not to find the ground state remains unchanged. For an easy sample with small $p_{f}$, all but a small fraction of the $n$ runs will end with a state of the same (namely the ground state) energy. For hard samples with larger $p_{f}$, however, states with different energies will result from a sizable fraction of runs, even if none of them is a ground state. In other words, the structure of low-lying excited states typically results in the appearance of a variety of energies for samples where a ground state is not found. By reacting to these events with additional runs for the affected samples until the same minimum energy has been found a certain number $n_{0}$ of times, the average failure probability $\left\langle p_{f}\right\rangle$ can be further decreased.

To demonstrate the power of this extension, $n=3$ runs of length $T=\mathcal{S}=32$ for 1000 samples of size $16 \times 16$ were performed. For 722 samples, all three runs ended with the same minimum energy, which was consequently ac- 
cepted as estimate for the ground-state energy. For the remaining replica, an additional run with $\mathcal{S}=32$ was performed, which settled 74 of the "questionable" cases. This scheme was iterated until for all 1000 samples the state of lowest energy had been found $n_{0}=3$ times. The average effort for this computation corresponded to $\mathcal{S} \approx 115$ (i.e., 3.6 runs of length $\mathcal{S}=32$ ), but the total number of missed ground states corresponded to that of runs with $\mathcal{S} \approx 240$ (or 7.5 runs of length $\mathcal{S}=32$ ). This type of computation can, of course, be favorably combined with the correlation results of Tab. I to perform a certain number of additional runs for samples where certain observable values indicate especially large failure probability $p_{f}$. Since the improvement effected by this addition depends on the structure of low-lying excited states of the model, it unfortunately cannot be quantified in general.

\section{CONCLUSIONS}

I have presented a novel optimization heuristic for finding numerically exact ground states of two-dimensional spin-glass systems with continuous spins with high reliability. Embedding Ising spins into the continuous rotators, this exponentially hard optimization problem is being related to the polynomial problem of finding Ising ground states on planar graphs via Edmonds' algorithm [43] for solving minimum-weight perfect matching problems. Due to a history dependence of effective coupling constants, this technique exhibits metastability on a low-energy subset of the metastable states of a single spin-flip zero-temperature quench. In contrast to simulated annealing and similar techniques, however, embedded matching has the crucial advantage of being strictly downhill in energy. To find true ground states, embedded matching is inserted as minimization procedure in a genetic algorithm specially tailored to the spin-glass ground-state problem. The essential component is here given by a properly chosen crossover operation exchanging automatically determined domains of rigidly locked spins between the parent replica, thus preserving the good optimization achieved at intermediate stages inside of domains and effectively allowing the method to directly operate on the manifold of metastable states.

This combination of techniques resulting in the genetic embedded matching (GEM) method outperforms general-purpose approaches such as simulated annealing by orders of magnitude: a success probability $p_{s} \geq 1 \%$ could not be achieved at all with reasonable computational effort with simulated annealing runs for the $16 \times 16$ bimodal $X Y$ samples considered for performance comparison. Due to the generally strong corrections to scaling present in spin-glass systems, the extension in accessible system sizes effected by the GEM approach over general-purpose techniques turns out to be crucial for the understanding of the asymptotic behavior of the spinglass phase, cf. Refs. [39, 40]. The distribution of success probabilities of the GEM technique can be understood from the decomposition theorem (12) of failure probabilities. The thus estimated distribution over disorder replica of minimum required runtimes or population sizes is perfectly described by a Fréchet distribution known from extreme-value theory, which is plausible given that sample hardness is determined by the hardest of a number of effective two-level systems describing the energy landscape of a disorder realization. Due to the heavy tail of this distribution, the exponential divergence of average computational effort with system size expected from non-polynomial optimization problems is accompanied by an increasing spread in sample hardness impeding an appropriate choice of optimization parameters common to all disorder samples. The variant approach ODGEM automatically maximizing genetic diversity by monitoring configurational overlap, reduces the severity of this spread by devoting additional effort to hard samples. Hard samples can also be detected by indicator observables of the disorder and low-energy configurations as well as the population in the genetic algorithm in order to decrease the failure probability in these cases. The decomposition property (12) finally allows to break up computations in smaller units which, besides allowing to further reduce the average effort required for a given success probability, makes the method ideally suitable for computations on parallel workstation and Beowulf clusters.

Note that for systems with degenerate ground states, the GEM and ODGEM methods as non-equilibrium techniques do not yield these different states with probabilities proportional to the corresponding Boltzmann factors, such that in these cases an additional postprocessing of the states found would become necessary 77]. Recent evidence suggests, however, that such degeneracies might be very unusual in disordered systems with continuous spins [39, 40]. The performance analysis presented here focused on the bimodal $X Y$ spin glass on the square lattice. The method straightforwardly generalizes to any other nearest-neighbor $\mathrm{O}(n)$ spin model (1) on planar graphs and to arbitrary disorder distributions. Specifically, the case of Gaussian bond distribution can be treated with similar efficiency. Due to the use of embedded matching as minimization component, the present from of the technique is limited to planar, two-dimensional lattices and the case of zero field. Other minimization techniques might be used in lieu of embedded matching inside of the genetic algorithm to tackle spin glasses in three dimensions or including magnetic fields.

\section{Acknowledgments}

I am indebted to M. Gingras for useful discussions and a critical reading of the manuscript. The research at the University of Waterloo was undertaken, in part, thanks to funding from the Canada Research Chairs 
Program (Michel Gingras). Some of the computations were performed at the facilities of the Shared Hierarchical Academic Research Computing Network (SHARC-
NET:www.sharcnet.ca). The author acknowledges support by the EC in form of a "Marie Curie Intra-European Fellowship" under contract No. MEIF-CT-2004-501422.
[1] D. J. Wales, Energy landscapes (Cambridge University Press, 2003).

[2] K. Binder and A. P. Young, Rev. Mod. Phys. 58, 801 (1986).

[3] A. K. Hartmann and H. Rieger, Optimization Algorithms in Physics (Wiley, Berlin, 2002).

[4] S. S. Plotkin and J. N. Onuchic, Quart. Rev. Biophys. 35, 111 (2002).

[5] D. A. Huse and D. S. Fisher, Phys. Rev. Lett. 57, 2203 (1986).

[6] P. Garstecki, T. X. Hoang, and M. Cieplak, Phys. Rev. E 60, 3219 (1999).

[7] S. Mertens, Comput. Sci. Eng. 4, 31 (2002).

[8] C. H. Papadimitriou, Computational Complexity (Addison-Wesley, Reading Mass., 1994).

[9] S. F. Edwards and P. W. Anderson, J. Phys. F 5, 965 (1975).

[10] F. Barahona, J. Phys. A 15, 3241 (1982).

[11] C. P. Bachas, J. Phys. A 17, L709 (1984).

[12] I. Bieche, R. Maynard, R. Rammal, and J. P. Uhry, J. Phys. A 13, 2553 (1980).

[13] L. Saul and M. Kardar, Phys. Rev. E 48, R3221 (1993).

[14] A. Galluccio, M. Loebl, and J. Vondrák, Phys. Rev. Lett. 84, 5924 (2000).

[15] F. Liers, M. Palassini, A. K. Hartmann, and M. Jünger, Phys. Rev. B 68, 094406 (2003).

[16] S. Kirkpatrick, J. Stat. Phys. 34, 975 (1984).

[17] B. A. Berg, U. E. Hansmann, and T. Celik, Phys. Rev. B 50, 16444 (1994).

[18] K. Hukushima and K. Nemoto, J. Phys. Soc. Jpn. 65, 1604 (1996).

[19] A. K. Hartmann, Physica A 224, 480 (1996).

[20] K. F. Pál, Physica A 223, 283 (1996).

[21] E. Marinari and G. Parisi, Phys. Rev. B 62, 11677 (2000).

[22] J. Houdayer and O. C. Martin, Phys. Rev. E 64, 056704 (2001).

[23] S. Boettcher and A. G. Percus, Phys. Rev. Lett. 86, 5211 (2001).

[24] J. J. Moreno, H. G. Katzgraber, and A. K. Hartmann, Int. J. Mod. Phys. C 14, 285 (2003).

[25] G. S. Grest, C. M. Soukoulis, and K. Levin, Phys. Rev. Lett. 56, 1148 (1986).

[26] N. Kawashima and H. Rieger, in Frustrated Spin Systems, edited by H. T. Diep (World Scientific, Singapore, 2005), chap. 9, p. 491

[27] C. Amoruso, A. K. Hartmann, M. B. Hastings, and M. A. Moore, Phys. Rev. Lett. 97, 267202 (2006).

[28] R. Fisch, J. Stat. Phys. 125, 789 (2006).

[29] A. Aromsawa and J. Poulter (2007), Preprint arXiv:0704.1186

[30] A. K. Hartmann (2007), Preprint arXiv:0704.2748.

[31] J. E. Greedan, J. Mater. Chem. 11, 37 (2001).

[32] P. A. Ferrari, A. Frigessi, and P. G. de Sá, J. Roy. Stat. Soc. B 57, 485 (1995).

[33] J. M. Kosterlitz and N. Akino, Phys. Rev. Lett. 82, 4094 (1999).
[34] L. R. Walker and R. E. Walstedt, Phys. Rev. B 22, 3816 (1980).

[35] B. W. Morris, S. G. Colborne, M. A. Moore, A. J. Bray, and J. Canisius, J. Phys. C 19, 1157 (1986).

[36] H. Kawamura and M. Tanemura, J. Phys. Soc. Jpn. 60, 608 (1991).

[37] J. Maucourt and D. R. Grempel, Phys. Rev. Lett. 80, 770 (1998).

[38] L. W. Lee and A. P. Young, Phys. Rev. E 72, 036124 (2005).

[39] M. Weigel and M. J. P. Gingras, Phys. Rev. Lett. 96, 097206 (2006).

[40] M. Weigel and M. J. P. Gingras (2007), Preprint arXiv:0706.0227

[41] G. Toulouse, Commun. Phys. 2, 115 (1977).

[42] A. Gibbons, Algorithmic Graph Theory (Cambridge University Press, Cambridge, 1985).

[43] J. Edmonds, J. Res. Natl. Bur. Stand. B 69, 125 (1965).

[44] W. Cook and A. Rohe, INFORMS J. Comput. 11, 138 (1999).

[45] J. W. Landry and S. N. Coppersmith, Phys. Rev. B 65, 134404 (2002).

[46] C. Amoruso, E. Marinari, O. C. Martin, and A. Pagnani, Phys. Rev. Lett. 91, 087201 (2003).

[47] U. Wolff, Phys. Rev. Lett. 62, 361 (1989).

[48] C. L. Henley, Ann. Phys. (NY) 156, 368 (1984).

[49] N. D. Mermin, Rev. Mod. Phys. 51, 591 (1979).

[50] G. Toulouse, Phys. Rep. 49, 267 (1979).

[51] M. Weigel and M. J. P. Gingras, J. Phys.: Condens. Matter 19, 145217 (2007).

[52] D. S. Fisher and D. A. Huse, Phys. Rev. Lett. 56, 1601 (1986).

[53] A. J. Bray and M. A. Moore, in Heidelberg Colloquium on Glassy Dynamics, edited by J. L. van Hemmen and I. Morgenstern (Springer, Heidelberg, 1987), p. 121.

[54] M. Mezard, G. Parisi, N. Sourlas, G. Toulouse, and M. Virasoro, J. Physique 45, 843 (1984).

[55] J. Houdayer and O. C. Martin, Europhys. Lett. 49, 794 (2000).

[56] C. L. Henley, Ann. Phys. (NY) 156, 324 (1984).

[57] J. Hoshen and R. Kopelman, Phys. Rev. B 14, 3438 (1976).

[58] Z. Michalewicz, Genetic Algorithms + Data Structures = Evolution Programs (Springer, Berlin, 1996).

[59] P. Sutton and S. Boyden, Am. J. Phys. 62, 549 (1994).

[60] U. Gropengiesser, J. Stat. Phys. 79, 1005 (1995).

[61] A. K. Hartmann, Phys. Rev. B 59, 3617 (1999).

[62] K. Pál, Biol. Cybern. 73, 335 (1995).

[63] M. Palassini and A. P. Young, Phys. Rev. Lett. 83, 5126 (1999).

[64] S. Kirkpatrick, C. D. Gelatt, and M. P. Vecchi, Science 220, 671 (1983).

[65] B. A. Berg and W. Janke, Phys. Rev. Lett. 80, 4771 (1998).

[66] S. Alder, S. Trebst, A. K. Hartmann, and M. Troyer, JSTAT p. P07008 (2004). 
[67] S. Geman and D. Geman, IEEE Trans. Pattern Anal. Mach. Intell. 6, 721 (1984).

[68] E. Castillo, Extreme Value Theory in Engineering (Academic Press, London, 1988).

[69] P. Dayal, S. Trebst, S. Wessel, D. Würtz, M. Troyer, S. Sabhapandit, and S. N. Coppersmith, Phys. Rev. Lett. 92, 097201 (2004).

[70] E. Bittner and W. Janke, Europhys. Lett. 74, 195 (2006).

[71] W. Feller, An Introduction to Probability Theory and its Applications, vol. 1 (John Wiley \& Sons, New York, 1968).

[72] M. A. Moore, Phys. Rev. Lett. 58, 1703 (1987).

[73] S. Kobe and T. Klotz, Phys. Rev. E 52, 5660 (1995).

[74] H. Kawamura, Phys. Rev. B 51, 12398 (1995).

[75] W. M. Saslow and G. Parker, Phys. Rev. Lett. 56, 1074 (1986).

[76] H. Kawamura, Phys. Rev. Lett. 68, 3785 (1992).
[77] A. K. Hartmann, Eur. Phys. J. B 13, 539 (2000).

[78] The product $\prod_{n} \Phi_{\square_{n}}$ over all plaquettes of the lattice is +1 for an even and -1 for an odd number of frustrated plaquettes. On the other hand, $\prod_{n} \Phi_{\square_{n}}=$ $\prod_{\langle i j\rangle}\left(\operatorname{sign} J_{i j}\right)^{2}=+1$, since each bond occurs twice in the product when taking into account external plaquettes for open boundaries.

[79] It is easy to see, for instance, that a pure Ising ground state $\boldsymbol{S}_{i}= \pm(1,0, \ldots)^{\mathrm{T}}, i=1, \ldots, L^{2}$ is invariant under the embedded matching algorithm as well as the local spin quench (2).

[80] Computationally, this enlargement of transformations is not very efficient since for inversions $\boldsymbol{S}_{i} \mapsto-\boldsymbol{S}_{i}$ the identification of frustrated plaquettes of the embedded Ising model depends on the configuration $\left\{\boldsymbol{S}_{i}\right\}$ of the $\mathrm{O}(n)$ spins, i.e., one finds $\operatorname{sign} \tilde{J}_{i j}^{r} \neq \operatorname{sign} J_{i j}$ in general. 